\title{
Microbial diversity of extreme habitats in human homes
}

\author{
Amy M Savage ${ }^{\text {Corresp.. }}{ }^{1}$, Justin Hills ${ }^{2}$ ， Katherine Driscoll ${ }^{3}$ ， Daniel J Fergus ${ }^{4}$ ，Amy M Grunden ${ }^{5}$, Robert R \\ Dunn ${ }^{6}$ \\ 1 Rutgers, The State University of New Jersey - Camden \\ ${ }^{2}$ Laboratory of Cellular and Molecular Biology, National Institute of Diabetes and Digestive and Kidney Diseases, Bethesda, MD, United States \\ 3 Animal Management Department, The Wilds, Cumberland, $\mathrm{OH}$, United States \\ ${ }^{4}$ Genomics and Microbiology, North Carolina Museum of Natural Sciences, Raleigh, NC, United States \\ 5 Department of Plant and Microbial Biology, North Carolina State University, Raleigh, NC, United States \\ 6 Department of Applied Ecology and Keck Center for Behavioral Biology, North Carolina State University, Raleigh, NC, United States \\ Corresponding Author: Amy M Savage \\ Email address: amy.savage@rutgers.edu
}

High-throughput sequencing techniques have opened up the world of microbial diversity to scientists, and a flurry of studies in the most remote and extreme habitats on earth have begun to elucidate the key roles of microbes in ecosystems with extreme conditions. These same environmental extremes can also be found closer to humans, even in our homes. Here, we used high-throughput sequencing techniques to assess bacterial and archaeal diversity in the extreme environments inside human homes (e.g., dishwashers, hot water heaters, washing machine bleach reservoirs, etc.). We focused on habitats in the home with extreme temperature, $\mathrm{pH}$, and chemical environmental conditions. We found a lower diversity of microbes in these extreme home environments compared to less extreme habitats in the home. However, we were nonetheless able to detect sequences from a relatively diverse array of bacteria and archaea. Habitats with extreme temperatures alone appeared to be able to support a greater diversity of microbes than habitats with extreme $\mathrm{pH}$ or extreme chemical environments alone. Microbial diversity was lowest when habitats had both extreme temperature and one of these other extremes. In habitats with both extreme temperatures and extreme $\mathrm{pH}$, taxa with known associations with extreme conditions dominated. Our findings highlight the importance of examining interactive effects of multiple environmental extremes on microbial communities. Inasmuch as taxa from extreme environments can be both beneficial and harmful to humans, our findings also suggest future work to understand both the threats and opportunities posed by the life in these habitats. 
1 Microbial diversity of extreme habitats in human homes

2 Amy M. Savage* 1, Justin L. Hills ${ }^{2}$, Katherine Driscoll ${ }^{3}$, Daniel J. Fergus ${ }^{4}$, Amy M. Grunden ${ }^{5}$,

3 Robert R. Dunn ${ }^{6}$

4 * Corresponding author: Amy.Savage@,rutgers.edu

5 Other authors: JLHills14@gmail.com, Katherine.Driscoll14@gmail.com,

6 DanielJFergus@gmail.com, amgrunde@ncsu.edu, RRDunn@ncsu.edu

7 1- Department of Biology \& Center for Computational and Integrative Biology, Rutgers

8 University, Waterfront Technology Center, 200 Federal Street, Camden, NJ 08102 08308322

3- Animal Management Department, the Wilds, 14000 International Road, Cumberland OH

2- Laboratory of Cellular and Molecular Biology, National Institute of Diabetes and Digestive and Kidney Diseases, 8 Center Drive, MSC 0830, Bethesda, MD $20892-$

4- Genomics and Microbiology, North Carolina Museum of Natural Sciences, 11 West Jones St, Raleigh, NC 27601

5- Department of Plant and Microbial Biology, North Carolina State University, 4550A Thomas Hall, Campus Box 7612, Raleigh, NC, 27695-7612

6- Department of Applied Ecology and Keck Center for Behavioral Biology, North Carolina State University, Campus Box 7617, Raleigh, NC 27695-7617 


\section{Abstract:}

28 High throughput sequencing techniques have opened up the world of microbial diversity to

29 scientists, and a flurry of studies in the most remote and extreme habitats on earth have begun to

30 elucidate the key roles of microbes in ecosystems with extreme conditions. These same

31 environmental extremes can also be found closer to humans, even in our homes. Here, we used

32 high throughput sequencing techniques to assess bacterial and archaeal diversity in the extreme

33 environments inside human homes (e.g., dishwashers, hot water heaters, washing machine bleach

34 reservoirs, etc.). We focused on habitats in the home with extreme temperature, $\mathrm{pH}$ and chemical

35 environmental conditions. We found a lower diversity of microbes in these extreme home

36 environments compared to less extreme habitats in the home. However, we were nonetheless

37 able to detect sequences from a relatively diverse array of bacteria and archaea. Habitats with

38 extreme temperatures alone appeared to be able to support a greater diversity of microbes than

39 habitats with extreme $\mathrm{pH}$ or extreme chemical environments alone. Microbial diversity was

40 lowest when habitats had both extreme temperature and one of these other extremes. In habitats

41 with both extreme temperatures and extreme $\mathrm{pH}$, taxa with known associations with extreme

42 conditions dominated. Our findings highlight the importance of examining interactive effects of

43 multiple environmental extremes on microbial communities. Inasmuch as taxa from extreme

44 environments can be both beneficial and harmful to humans, our findings also suggest future

45 work to understand both the threats and opportunities posed by the life in these habitats. 
46 Keywords: Community Structure, Extreme environments, Human Homes, Interactive effects,

47 Microbial Diversity

Introduction:

52 The innovation of culture-independent, high-throughput sequencing techniques has facilitated the

53 discovery of high microbial diversity in many habitats once considered inhospitable to life

54 (Rothschild and Mancinelli 2001). The species in these environments are frequent targets for the

55 discovery of useful enzymes (Niehaus et al. 1999, van den Burg 2003, Elleuche et al. 2014), and

56 studies of microbes living in extreme environments have provided key insights into the evolution

57 of microbial metabolism (Valentine 2007, Hoehler and Jorgensen 2013). Often overlooked,

58 however, is that the attributes that define many of the most extreme habitats on Earth, such as

59 extremes of temperature, $\mathrm{pH}$, water activity, or low nutrient levels, can also be found more

60 immediate to everyday experience. Human homes, for example, contain microhabitats as hot,

61 acidic, basic or salty as any encountered elsewhere on Earth (Martin et al. 2015).

62 We know of only two extreme habitats within homes where microbial diversity has been studied

63 to date, and in both cases culture-dependent techniques were used. In 1973, Brock and Boylen

64 discovered a species of the genus Thermus (T. aquaticus) living in hot water heaters. Species of

65 this genus had previously been known only from hot springs (Brock and Boylen 1973). In

66 addition, studies have considered the biology of tap water. Tap water is hospitable in terms of its

67 abiotic conditions (e.g. temperature, $\mathrm{pH}$, toxicity) but is very low in nutrients and so was long 
68 assumed to be relatively devoid of life; until, that is, it was studied. Tap water has now been

69 shown to contain many species of bacteria capable of surviving in low nutrient environments

70 (Kalmbach et al. 1997, Szewzyk et al. 2000, Boe-Hansen et al. 2002). If life exists in hot water

71 heaters and tap water, it seems possible and even likely that many extreme habitats in homes

72 sustain life. That the environmental extremes imposed by these conditions in homes (cold, hot,

73 acidic, alkaline, wet or dry) delineate which species are present seems inevitable. That they are

74 lifeless is unlikely.

75 Here, we used culture-independent, high-throughput sequencing to address the following

76 questions: (1) What is the relative diversity of microbes (specifically, Bacteria and Archaea)

77 under extremes of temperature, $\mathrm{pH}$ and chemical environments of southeast US homes and how

78 does it compare to habitats without each extreme conditions? Harrison et al. (2013) recently

79 argued that because many extreme environments include simultaneous extremes in multiple

80 environmental factors, interactive effects of these multiple sources of extreme conditions are

81 likely to be important determinants of microbial diversity in extreme environments. Therefore,

82 we additionally asked (2) how do multiple, simultaneous extreme conditions influence microbial

83 diversity in human homes? Finally, we asked (3) which bacterial and archaeal genera from the

84 broader home (Dunn et al. 2013) fail to persist in extreme home habitats, and which microbial

85 genera persist only in these extreme habitats?

\section{Methods:}

\section{Sampling extreme home environments}

88 We sampled extreme environments in six houses in the Raleigh-Durham metropolitan area

89 (Supp. Fig 1). In each house, we used dual-tipped sterile BBL ${ }^{\mathrm{TM}}$ CultureSwabs ${ }^{\mathrm{TM}}$ or $50 \mathrm{ml}$ 
90 conical tubes to collect water from each of 10 standardized extreme locations in homes. The sites

91 sampled in all six houses included environments that were extreme in terms of their temperature,

$92 \mathrm{pH}$ and/or chemical environments (Supp. Table 1). Our assumptions concerning these sampling

93 locations are based upon publicly available consumer resources regarding certain commercial

94 and industrial requirements (e.g. http://www.nsf.org/consumer-resources/health-and-safety-

95 tips/home-product-appliance-tips/sanitizing-dishwasher,

96 http://energy.gov/energysaver/projects/savings-project-lower-water-heating-temperature). For

97 example, our sampling of dishwashers was influenced by the NSF/ANSI 184 standard for

98 residential dishwashers to provide a final rinse at a temperature of at least $150{ }^{\circ} \mathrm{F}\left(65.6{ }^{\circ} \mathrm{C}\right)$.

Additionally, temperature ranges for residential water heaters are 90 to $150 \mathrm{~F}\left(32-65.6^{\circ} \mathrm{C}\right)$,

100 depending on the manufacturer. Bleach receptacles in clothes washing machines would also be

101 assumed to have a $\mathrm{pH}$ of 12 when bleach is present. Although the $\mathrm{pH}$ and chemical composition

102 of laundry detergent and dishwasher detergent can be quite variable, manufacturing standards are

103 generally within the 7-10 $\mathrm{pH}$ range. While measurements, opposed to assumptions, would be

104 very useful, taking measurements of all the potential extreme axes under various sample sites in

105 multiple homes was not feasible. All samples were preserved at $-20^{\circ} \mathrm{C}$ immediately after

106 collection.

107 Isolating and identifying microbes in extreme home environments

108 Genomic DNA was extracted from all samples using the MoBio Power Soil DNA extraction kit

109 (MoBio, Carlsbad, CA) as described previously (Fierer et al., 2008; Lauberet al., 2009). For

110 swabs, the tips were placed in PowerBead tubes containing solution $\mathrm{C} 1$ and swirled vigorously

111 for approximately 10 seconds to release contents and removed. Water samples were thawed and

112 filtered using Corning $50 \mathrm{ml} 0.22 \mathrm{um}$ cellulose acetate filters after which the filters were added to 
113 the PowerBead tubes. The extractions were subsequently performed as directed by the

114 manufacturer, except that the final elution was performed in $50 \mu 1$ of $70^{\circ} \mathrm{C}$ C6 elution buffer.

115 Because the water samples were frozen prior to filtering and extraction, the results reported for

116 the water samples likely under-represents the true diversity of taxa in those environments.

117 We used methods described in Bates et al (2011) to amplify bacterial and archaeal DNA from the

118 samples collected from homes and six negative controls. Briefly, amplicons were produced by

119 PCR with universal bacterial/archaeal 515F and 806R primers to which Roche $454 \mathrm{~B}$

120 pyrosequencing adapters had been added, as described in Hulcr et al. (2012). The 515F primer

121 contained an additional 12-bp barcode sequence for individual sample identification. All the

122 samples were amplified by triplicate PCR reactions, cleaned using the UltraClean-htp 96-well

123 PCR Clean-up kit (MoBio), and quantified with a Quant-iT PicoGreen dsDNA Assay kit

124 (Invitrogen). Equimolar amounts of each sample were pooled into a single sample to sequence.

125 DNA pyrosequencing was performed at Selah Clinical Genomics Center at Innovista (University

126 of South Carolina, USA) using a Roche Genome Sequencer 454 FLX system to facilitate

127 comparison to previous related work that utilized this platform (Dunn et al, 2013). Though these

128 methods here do not distinguish living from recently dead cells, with the comparative approach

129 used here, we presume that taxa frequently identified in one habitat but rare or absent in most

130 others are likely surviving in the habitat from which they are frequently identified. The

131 sequences were submitted to NCBI (SRA accession number SRP071677).

132 The QIIME analysis package (Caporaso et al, 2010a) was used to process and analyze the

133 barcoded microbial (bacterial and archaeal) amplicon sequences. Sequences were quality filtered

134 to a minimum quality score of 25 with no unambiguous bases and sorted to each sample by the

13512 bp barcodes. The 454 pyrosequencing produced 197,305 reads that passed the quality 
136 screening. The sequences were grouped into Operational Taxonomic Units (OTUs) that shared at

137 least $97 \%$ sequence similarity. A representative sequence was taken for each OTU group and

138 PyNAST (Caporaso et al, 2010b) was employed to align these representative sequences to the

139 Greengenes database (DeSantis et al, 2006) and the taxonomic identity of each OTU was

140 determined using the RDP Classifier (Wang et al, 2007). Phylotypes were considered to be

141 contaminants if they were seen in at least two of the six negative control samples. There were

142152 OTUs at the genus level present in more than one negative sample, representing $9 \%$ of the

143 total OTUs at this level. After removing contaminant sequences and singletons, the number of

144 quality-filtered reads per sample was between 6 and 5861 (median=2306). Finally, we removed

145 any OTU's represented by 20 or fewer reads to reduce the possibility of spurious results from the

146 sequencing process. For among samples comparisons we rarefied to each to a depth of 1000

147 sequences. Thus, our assessments of microbial diversity are conservative.

Analysis of the relative diversity of microbes in extreme temperature, $\mathrm{pH}$ and chemical environments of homes and how it compares to habitats without each extreme condition

151

152

153

154

155

156

157

158

We compared microbial species accumulation among three extreme variables in homes: temperature, $\mathrm{pH}$, and chemical extremes. Temperature was classified on a scale of 1-5, with 1 representing the coldest environments and 5 representing the hottest environments. We then binned 1 and 5 into an extreme temperature category and 2-4 into an intermediate temperature category. Similarly, environments were classified as acidic, basic or neutral and then binned into extreme $\mathrm{pH}$ (acidic or basic environments) versus neutral environments. Finally, chemical extremes were those environments characterized by the presence of detergent, bleach, metals, ammonia, or natural gas (Supp. Table 2). 
159 We used EstimateS v. 9.1.0 (Colwell 2013) to construct individual-based species accumulations

160 for all three extreme environments and their non-extreme counterparts. For these curves, reads

161 were used as individuals and the curves were constructed using 1000 iterations. To formally

162 assess differences in accumulated species by read, we used $\pm 95 \%$ confidence intervals for each

163 curve. Non-overlapping 95\% confidence intervals are considered formal evidence of significance

164 (Knezevic 2008).

165 Assessing how multiple, simultaneous extreme conditions influence microbial diversity in human 166 homes

167 We were interested in testing the hypothesis that interactive effects of multiple, simultaneously 168 extreme environmental conditions are important determinants of microbial diversity in extreme 169 home environments (Harrison et al. 2013). Our study included multiple samples with more than 170 one environmental extreme (Supp. Table 1); however, we only had sufficient replication to 171 assess this hypothesis for 2-way interactions between extremely high temperatures and extreme

$172 \mathrm{pH}$ as well as high temperature and chemical environments. Because number of reads varied

173 significantly among different environmental extremes, we could not use a standard 2-way

174 ANOVA. Instead, we assessed these effects using an ordination framework.

175 We visualized the composition of bacteria and archaea from extreme habitats in homes using

176 non-metric multidimensional scaling ordination (NMDS) in Primer-E v.7.0.9 with

177 PERMANOVA +1 (Clarke \& Gorley, 2015). To do this, we first constructed NMDS plots with

178100 restarts and a Type I Kruskal fit scheme based on a Dissimilarity matrix of Bray-Curtis

179 distances. To assess the relationship between temperature (extreme $v s$. intermediate) and the 180 other extremes ( $\mathrm{pH}$ : extreme $v s$. neutral; chemicals: extreme $v s$. none) for a-diversity of 181 microbes, we conducted a permuted multivariate analysis of variance (PERMANOVA) test with 
182 temperature class and either $\mathrm{pH}$ or chemical class and their interaction as factors, 9,999 iterations

183 and Type III sums of squares. When interactions were significant (Anderson et al. 2008), we

184 conducted pairwise PERMANOVA to determine which treatment combinations significantly

185 differed from one another. Similarly, we assessed these relationships in terms of $\beta$-diversity

186 using a permuted dispersion (PermDisp) test of a presence/absence matrix of OTU occurrences.

187 When these tests were significant, we conducted pairwise tests of extreme $v s$. non-extreme

188 chemical and $\mathrm{pH}$ environments in habitats with intermediate and extreme temperatures (thus 2

189 tests per treatment combination). Finally, we conducted SIMPER analyses for each significant

190 treatment combination to determine the OTUs that contributed the most to pairwise between-

191 group differences in ordination space. Because we conducted two separate analyses for each

192 level of diversity, we accounted for the additional error associated with multiple tests, using a

193 revised $a=0.05 / 2=0.025$ as our cut-off for statistical significance for the results of each test. This

194 conservative a is particularly important because we did not have equal sample sizes in all groups

195 for these analyses, which can increase the risk of Type I error (Anderson \& Walsh 2013).

Determining which microbial genera differentiate extreme home habitats from the rest of the

198 home

We compared the occurrences of microbes in our samples to those reported in less extreme home environments (Dunn et al. 2013). Human-associated microbes were common to both datasets, and we were particularly interested in those taxa unique to our dataset, relative to the broader

202 home (Dunn et al. 2013). Therefore, we removed human-associated OTU's from our dataset. We identified these human-associated OTU's using databases that identified human gut (Flores et al.

204 2014) and skin (Urban et al. 2016) microbiomes. OTU's that occurred in at least 80\% of the 
205

206

207

208

209

210

211

212

213

214

215

216

217

218

219

220

221

222

223

224

225

226

samples in those databases were considered human-associates and excluded from our analyses of the microbial diversity of extreme habitats in human homes. We then determined the identity of microbes that were absent from the broader homes dataset, but present in extreme environments and then tabulated the extreme habitat(s) in which they were present. Likewise, we identified the non-human associated microbes that were present in the broader home environment, but absent from all extreme environments in our samples.

\section{Results and Discussion:}

What is the relative diversity of microbes in extreme temperature, $p H$ and chemical environments of southeast US homes and how does it compare to habitats without each extreme condition?

The cumulative diversity (OTU richness) in habitats with extreme temperatures was more than twice as high as in habitats with extreme $\mathrm{pH}$ (maximum of $73 \mathrm{vs.} 33$, Fig. 1) and almost three times as high as habitats with extreme chemical environments (27.6; Fig. 1). Habitats with extreme temperatures also had higher OTU richness than habitats with intermediate temperatures (Fig. 2a). Conversely, previous research indicates that the diversity in habitats with either extremely high or extremely low temperatures is generally low, and dominated by a small number of abundant bacterial species (Lewin et al. 2013). For example, Sharp et al. (2014) recently found that OTU richness in hydrothermal vents peaked at intermediate temperatures $\left(24^{\circ} \mathrm{C}\right)$, with reduced OTU richness in extremely hot or cold environments (Sharp et al. 2014).

We did not detect significant differences in the rarefied species richness of bacterial and archaeal microbes in extreme $v s$. neutral $\mathrm{pH}$ conditions; however, the marginally non-significant trend suggests that extreme $\mathrm{pH}$ environments also had higher microbial diversity than neutral habitats 
227 (Fig. 2b). Recent studies have demonstrated that $\mathrm{pH}$ is a key predictor of microbial diversity in

228 both extreme environments, such as acid mine drainage sites (Kuang et al. 2013), and less

229 extreme environments, such as tropical soils (Tripathi et al. 2012). In both cases, habitats with

230 neutral $\mathrm{pH}$ had higher microbial diversity than those with a $\mathrm{pH}$ higher or lower than neutral.

231 Thus, we again found different patterns in extreme home environments compared to other studies

232 comparing extreme and non-extreme habitats. One possible explanation for the difference

233 between our findings and these recent studies is that human-associated microbes are present in

234 home environments with intermediate temperatures. Perhaps these species are able to dominate

235 habitats with intermediate, but not extreme, conditions. Alternatively, the lower diversity in

236 habitats with intermediate temperatures and neutral $\mathrm{pH}$ in our study could be due to the

237 occurrence of extreme conditions along different axes (e.g. intermediate temperature, but

238 extreme $\mathrm{pH}$ or chemical habitats). We examine potential interactive effects of these polyextreme

239 habitats in the next section.

In contrast, habitats with extreme chemicals had significantly lower accumulated OTU

241 richness than did habitats without these extreme conditions (Fig. 2c). Extreme chemical

242 environments are poorly studied and understood (Rothschild and Mancinelli 2001). Our data

243 suggest that they could act as strong filters in extreme environments.

244 How do multiple, simultaneous extreme conditions influence microbial diversity in extreme home

245 environments?

Many of the habitats in this study were characterized by more than one extreme

247 environmental condition. Therefore, we also examined the potential for interactive effects of

248 multiple, simultaneous extreme conditions on microbial diversity. Due to limited replication

249 across all environmental extremes, we were only able to examine extreme $\mathrm{pH}$ and chemical 
250 habitats with and without extreme temperatures. We used an ordination framework to examine

251 these interactive effects (see methods).

252 We found significant interactions between extreme temperature and both extreme $\mathrm{pH}$

253 (PERMANOVA: Pseudo- $F_{1,82}=2.53, P=0.0001$; Figure $3 \mathrm{~A}$ ) and extreme chemical

254 (PERMANOVA: Pseudo- $F_{1,82}=3.16, P=0.0001$; Figure 3C) environments for OTU

255 composition. When temperatures were intermediate, there were no significant differences in

256 microbial composition in extreme vs. neutral $\mathrm{pH}$ habitats (pairwise PERMANOVA: $\mathrm{t}_{1,38}=1.02$,

$257 P=0.40)$. However, when temperatures were extreme, there was a very large difference between

258 the composition of microbes in extreme $\mathrm{pH}$ habitats, compared to neutral habitats (pairwise

259 PERMANOVA: $\mathrm{t}_{1,38}=1.70, P=0.0001$; Fig. $\left.3 \mathrm{~A}\right)$. The five genera that contributed the most to

260 differences between these two habitat types (from SIMPER analysis) were Parascardovia,

261 Micrococcus, Rothia, Brachybacterium, and an unknown genus from Sphingomonadaceae. Most

262 of these genera are associated with humans (Oshima et al. 2015, Gueimonde et al. 2012, Kloos et

263 al. 1975, Kocur et al. 2006, Vaccher et al. 2007, Uchibori et al. 2012). Sphingomonadaceae are

264 widespread in aquatic habitats, including drinking water (Vaz-Moreira et al. 2011), but also other

265 aquatic environments (e.g. tree holes-Xu et al. 2008). Brachybacterium is usually associated with

266 marine environments (Ward and Boru 2006), including Antarctic sea ice (Junge et al. 1998).

267 However, it was recently detected in an urban shopping center (Tringe et al. 2008). All of these

268 genera were more common in habitats with extreme temperatures and neutral $\mathrm{pH}$ than they were

269 in habitats with both extreme temperatures and extreme $\mathrm{pH}$. While different houses had

270 significantly different microbial composition (3-way PERMANOVA, $\mathrm{P}=0.0001$ ), there were no

271 significant 2-or 3-way interactions with house (Supp. Table 3). 
272 The interaction between temperature and chemical extremes was similar. Microbial composition

273 was indistinguishable between the habitats that only had one extreme condition-regardless of

274 whether it was temperature or chemicals that were extreme. There were also no significant

275 differences between habitats with neither extreme temperatures nor extreme chemical conditions

276 and habitats that had a single extreme condition. However, habitats with both extreme

277 temperatures and extreme chemicals had significantly different microbial composition compared

278 to all other groups (pairwise PERMANOVA; $\mathrm{t}_{1,38}=1.75, P=0.0001$; Fig. 3C). The five genera

279 that contributed the most to compositional difference between these two habitats (from SIMPER

280 analysis) were Methylobacterium, an unknown genus of Moraxellaceae, Sejonia, an unknown

281 genus of Sphingomonadaceae, and Flavobacterium. With the exception of the unknown genus

282 of Moraxellaceae, which was more common in extreme chemical and temperature environments,

283 all of these genera were more common in the habitats without temperature and chemical

284 extremes. Moraxallaceae have been found in other extreme environments, including deep sea

285 sediments (Maruyama et al. 1997). Although it was more common in our less extreme

286 environments, Sejonia is better known from Antarctic ice (Yi et al. 2005). Sphingomonadaceae

287 as described above are common to aquatic habitats. Methylobacterium is a widespread habitat

288 generalist that is facultatively methyltrophic (Green 2006). Finally, Flavobacterium is common

289 in freshwater and marine ecosystems but tends to flourish in cold environments with high salinity

290 (Bernardet and Bowman 2006).

291 There were also significant differences in the $\beta$-diversity in home environments with more than

292 one extreme condition. When temperatures were intermediate, there were no significant

293 differences between neutral and extreme $\mathrm{pH}$ environments (Figure 4A; PermDisp: $P=0.3864$ ).

294 However when temperatures were also extreme, habitats with extreme $\mathrm{pH}$ conditions had 
295 significantly higher $\beta$-diversity than those with neutral $\mathrm{pH}$ conditions (Figure 4A; PermDisp:

$296 P=0.0014)$. Similarly, at intermediate temperatures, there was a non-significant trend (Figure 4B;

297 PermDisp: $P=0.03$, Bonferroni-corrected $a=0.025)$ in which habitats without extreme

298 chemicals present had higher $\beta$-diversity than those with extreme chemicals present. However,

299 when temperatures were also extreme, habitats with extreme chemicals present had higher $\beta$ -

300 diversity than those without extreme chemicals (Figure 4B; PermDisp: $P=0.0006$ ). This

301 increase in $\beta$-diversity in extreme $\mathrm{pH}$ and chemical environments when temperatures were also

302 extreme suggests that polyextreme conditions may support a higher diversity of extremophiles

303 and/or reduced occurrences of numerically dominant genera compared to environments with a

304 single extreme condition, at least among habitats (in contrast to within habitats). The 5 genera

305 that contributed the most to differences in $\beta$-diversity between neutral and extreme $\mathrm{pH}$ conditions

306 when temperatures were also extreme were: Veillonella, Kocuria, Peptoniphilus, Parascardovia,

307 and Anaerococcus. Interestingly, these were also the top 5 genera contributing to differences

308 between habitats with and without extreme chemicals that also had extreme temperatures. All of

309 these genera were less common in habitats with 2 extremes than they were in habitats with only

310 extreme temperatures. They are also genera that include human-associated species (Bhatti \&

311 Frank 2000, Fadda et al. 2001, Song et al. 2007, Gueimonde et al. 2012).

Which microbial genera differentiate extreme home habitats from the rest of the home?

After removing all human-associated microbes (see methods), there were a total of 241 unique

315 genera in the broader homes dataset (Dunn et al. 2013). Our extreme samples contained 135 of

316 the remaining broader homes genera, but $\sim 44 \%$ of the genera found in the broader homes were

317 absent from our extreme home samples (Supp. Table 4), the absence of which might simply be 
318 due to the larger number of samples in Dunn et al. (2013). More interestingly, we found 20

319 genera present among our samples that were absent from the broader homes dataset. Nine of

320 these genera were found in all three categories of extreme environments (Table 1); one genus

321 (Solibacter) was absent from habitats with extreme $\mathrm{pH}$, but occurred in both extreme chemical

322 and temperature environments. Solibacter is a common and abundant soil microbe, especially in

323 tropical regions (Guan et al. 2013, Wang et al. 2015). There was also one genus

324 (Brevundimonas) that was absent from extreme chemical environments, but present in both

325 extreme temperature and extreme $\mathrm{pH}$ environments; Brevundimonas is one of the only genera

326 thought to be able to survive the low temperatures and ionizing radiation on Mars (Dartnell et al.

327 2010). There were three genera (Azobacteroides, Elizabethkingia, and Xiphinematobacter) that

328 occurred in both extreme $\mathrm{pH}$ and chemical environments that were absent in extreme

329 temperature environments. Both Azobacteroides and Xiphinematobacter are gut symbionts of

330 invertebrates; Azobacteroides is commonly found inside the protozoan symbionts of termites

331 (Noda et al. 2007), and Xiphinematobacer is an endosymbiont of nematodes (Vandekerckhove et

332 al. 2000). In invertebrate guts these microbes likely experience extreme chemical and $\mathrm{pH}$

333 environments frequently, while being relatively protected from temperature stress.

334 Elizabethkingia is a cosmopolitan genus, with species that are endosymbionts of mosquitoes

335 (Kämpfer et al. 2011), and others that are pathogens of both humans (Ceyhan and Celik 2011)

336 and frogs (Xie et al. 2009). There was one genus that was only found in extreme chemical

337 environments (Helcococcus). Interestingly, members of the genus Helcococcus possess the

338 ability to degrade detergents. In fact, the detergent Tween- 80 can be added to media to enrich

339 Helcococcus (Collins et al. 1993, Chagla et al. 1998). Finally, we found 5 genera (Brochothrix,

340 Buchnera, Polynucleobacter, Ralstonia, and Thermicanus) unique to extreme temperature 
341 environments. Brochothrix is a common spoilage bacterium in meat (Rattanasomboom et al.

342 1999). Buchnera is a widespread aphid endosymbiont (Shigenobu et al. 2000). Recently, a

343 survey of homes in Raleigh, NC demonstrated that aphids could be quite common in human

344 homes (Bertone et al. 2016), which could explain how this genus arrived in the homes in our

345 study (via aphids in the home). The genus Polynucleobacter includes both free-living species and

346 species that are endosymbionts of nematodes (Vannini et al. 2007). Ralstonia metallidurans is a

347 bacterium specifically adapted to toxic metal environments (Mergeay et al. 2003). Other species

348 of Ralstonia have been shown to be effectively controlled using high temperature treatments in

349 commercial crops (Kongkiattikajorn and Thepa 2007). In our study, Ralstonia were collected in

350 both high and low temperature environments. Finally, Thermicanus is, as its name suggest, a

351 thermophilic bacterial genus (Wrighton et al. 2008).

\section{Conclusions:}

353 This study has provided a glimpse into the microbial diversity that lives in habitats of human

354 homes similar in their extreme temperature, $\mathrm{pH}$ and chemical conditions to some of the most

355 extreme habitats on Earth. We discovered that these conditions have lower diversity than the

356 surrounding home environment; yet tens of bacterial lineages can be found in these extreme

357 habitats of the human home, including many taxa with known associations with extreme

358 conditions. Habitats with extreme temperatures alone appear to be able to support a greater

359 diversity of microbes than habitats with extreme $\mathrm{pH}$ or extreme chemical environments alone.

360 Microbial diversity is significantly lowest when habitats have both extreme temperature and one

361 of these other extremes. Interestingly, environments in homes often alternate between periods of

362 extreme and non-extreme conditions. For example, dishwashers are only likely to have extremely

363 high temperatures while cleaning and drying dishes. This variability could lead to temporal shifts 
364 in microbial composition, similar to those found for human vaginal microbes (Gajer et al. 2012).

365 This variability may also explain the presence of human-associated generalist species in our

366 samples. Future work, with samples taken before and after appliances (like many of those used in

367 our study) are operated, could elucidate the importance of episodic extreme conditions for

368 microbial communities in homes. Additionally, a key next step is understanding which of the

369 relatively few species that are found in these poly-extreme environments in the home are

370 metabolically active there and both whether these polyextreme taxa pose health threats (as was

371 recently suggested by Gümral et al. 2015) and/or might be useful industrially.

Acknowledgements: We thank the homeowners for allowing us to sample habitats in their

homes. Thanks also to Dr. Holly Menninger for her support and guidance in addressing logistical challenges presented by the current work. The Genomics Laboratory at the North Carolina Museum of Natural Sciences provided critical logistical support for this project.

377

\section{References}

Anderson, MJ, Gorley RN, Clark, KR (2008) PERMANOVA+ for PRIMER: Guide to software and statistical methods. PRIMER-E: Plymouth, UK.

Bates ST, Berg-Lyons D, Caporaso JG, Walters WA, Knight R, Fierer N (2011) Examining the global distribution of dominant archaeal populations in soil. ISME J 5: 908.

Bertone MA, Leong M, Bayless KM, Malow TLF, Dunn RR, Trautwein MD (2016) Arthropods of the great indoors: characterizing diversity inside urban and suburban homes. PeerJ 4:e1582 https://doi.org/10.7717/peerj.1582. 
385 386

387

388

389

390

391

392

393

394

395

396

397

398

399

400

401

402

403

404

405

Boe-Hansen R, Albechtsen H-J, Arvin E, Jorgensen Claus (2002) Bulk water phase and biofilm growth in drinking water at low nutrient conditions. Water Research 36: 4477-4486.

Brock TD, Boylen KL (1973) Presence of thermophilic bacteria in laundry and domestic hot water heaters. Applied Microbiology 25: 72-76.

Caporaso JG, Kuczynski J, Stombaugh J, Bittinger K, Bushman FD, Costello EK, Fierer N, Peña AG, Goodrich JK, Gordon J , Huttley GA, Kelley ST, Knights D, Koenig JE, Ley RE, Lozupone CA, McDonald D, Muegge BD, Pirrung M, Reeder J, Sevinsky JR, Turnbaugh PJ, Walters WA, Widmann J, Yatsunenko T,Zaneveld J, Knight R. (2010a) QIIME allows analysis of high-throughput community sequencing data. Nature Methods 7: 335-336.

Caporaso JG, Bittinger K, Bushman FD, DeSantis TZ, Andersen GL, and Knight R. (2010b) PyNAST: a flexible tool for aligning sequences to a template alignment. Bioinformatics 26: 266-267.Ceyhan M and Celik M (2011) Elizabethkingia meningosepticum (Chryseobacterium meningosepticum) infections in children. International Journal of Pediatrics. doi:10.1155/2011/215237/

Chagla AH, Borczyk AA, Facklam RR, Lovgren M. (1998) Breast abscess associated with Helcococcus kunzii. Journal of Clinical Microbiology 36:2377-2379.

Collins MD, Facklam RR, Rodrigues UM, Ruoff KL (1993) Phylogenetic analysis of some Aerococcus-like organisms from clinical sources: description of Helcococcus kunzii gen. nov., sp. nov. Int. J. Syst. Bacteriol. 43:425- 429.

Colwell RK (2013) EstimateS: Statistical estimation of species richness and shared species from samples. Version 9. Persistent URL < purl.oclc.org/estimates> 
406

407

408

409

410

411

412

413

414

415

416

417

418

419

420

421

422

423

424

425

426

Dartnell LR, Hunter SJ, Lovell KV, Coates AJ, Ward JM (2010) Low temperature ionizing radiation resistance of Deinococcus radiodurans and Antarctic Dray Valley bacteria. Astrobiology 7: 717-732.

DeSantis, TZ, Hugenholtz P, Larsen N, Rojas M , Brodie EL, Keller K, Huber T, Dalevi D, Hu P, and Andersen GL. (2006) Greengenes, a Chimera-Checked 16S rRNA Gene Database and Workbench Compatible with ARB. Applied Environmental Microbiology 72:5069-72.

Dunn RR, Fierer N, Henley JB, Leff JW, Menninger HL (2013) Home life: factors structuring the bacterial diversity found within and between homes. PLoS One. DOI: 10.1371/journal.pone.0064133

Elleuche S, Schröder C, Sahm K, Antranikian G. (2014) Extremozymes-biocatalysts with unique properties from extremophilic microorganisms. Current Opinion in Biotechnology 29: 116123.

Fierer N, Hamady M, Lauber CL, Knight R. (2008) The influence of sex, handedness, and washing on the diversity of hand surface bacteria. PNAS 105:17994-17999.

Flores GE, Caporaso JG, Henley JB, Rideout JR, Domogala D, Chase J, Leff JW, VásquezBaeza Y, Gonzalez A, Knight R, Dunn RR, Fierer N (2014) Temporal variability is a personalized feature of the human microbiome. Genome Biology 15: art531

Gajer P, Brotman RM, Bai G, Sakamoto J, Schütte UME, Zhong X, Koenig SK, Fu L, Ma Z, Zhou X, Abdo Z, Forney LJ, Ravel, J. (2012). Temporal Dynamics of the Human Vaginal Microbiota.Science Translational Medicine, 4(132), 132 ra52. doi.org/10.1126/scitranslmed.3003605 
427 Guan X, Wang J, Zhao H, Wang J, Luo X, Zhao F (2013) Soil bacterial communities shaped by 428 geochemical factors and land use in a less-explored area, Tibetan Plateau. BMC Genomics 14: 820 (13pp).

430

431

432

433

434

435

436

437

438

439

440

441

442

443

444

445

446

Gueimonde M, Bottacini F, van Sinderen D, Ventura M, Margolles A, Sanchez B (2012) Genome sequence of Parascardovia denticolens IPLA 20019, isolated from human breast milk. Journal of Bacteriology 194: 4776-4777.

Gümral R, Özhak-Baysan, Tümgör A, Saraçli MA, Yidrian ŞT, Ilkit M, Zupančič J, NovakBabič M, Gunde-Cimerman N, Zalar P, de Hoog GS (2015) Dishwashers provide a selective extreme environment for human-opportunistic yeast-like fungi. Fungal Diversity 10.1007/s13225-015-0327-8.

Harrison JP, Gheeraert N, Tsigelnitskiy D, Cockell CS (2013) The limits of life under multiple extremes. Trends in Microbiology 21: 204-212.

Hoehler TM, Jorgensen BB. (2013) Microbial life under extreme energy limitation. Nature Reviews Microbiology 11: 83-95.

Hulcr J, Latimer AM, Henley JB, Rountree NR, Fierer N, Lucky A, Lowman MD, Dunn RR (2012) A jungle in there: Bacteria in belly buttons are highly diverse, but predictable. PLoS One 7(11): e47712.

Junge K, Gosink JJ, Hoppe HG, Staley JT (1998) Arthrobacter, Brachybacterium and Planococcus isolates identified from Antarctic sea ice brine. Systematic and Applied Microbiology 21: 306-314. 
447 Kalmbach S, Manz W, Szewzyk U (1997) Isolation of new bacterial species from drinking water 448 biofilms and proof of their in situ dominance with highly specific 16S rRNA probes. Applied and Environmental Microbiology 63: 4164-4170.

450

451

452

453

454

455

456

457

458

459

460

461

462

463

464

465

Kämpfer P, Matthews H, Glaeser SP, Martin K, Lodders N, Faye I (2011) Elizabethkingia anopheles sp. nov., isolated from the midgut of the mosquito, Anopheles gambiae. International Journal of Evolutionary Microbiology. doi: 10.1099/ijs.o68147-0.

Kloos WE, Musselwhite MS (1975) Distribution and persistence of Staphylococcus and Micrococcus species and other aerobic bacteria on human skin. Applied Microbiology 30: $381-395$.

Knezevic A. (2008) Overlapping confidence intervals and statistical significance (http://www. cscu.cornell.edu/news/statnews/stnews73.pdf). In: Cornell University, Cornell Statistical Consulting Unit.

Kocur M, Koos WE, Schleifer KH (2006) The genus Micrococcus. Prokaryotes 3: 961-971.

Kongkiattikajorn J, Thepa S (2007) Increased tomato yields by heat treatment for controlling Ralstonia solanacearum in soil. Proceedings of the $45^{\text {th }}$ Kasetsart University Annual Conference.

Kuang J-L, Huang L-N, Chen L-X, Hua Z-S, Li S-J, Shu, W-S (2013) Contemporary environmental variation determines microbial diversity patterns in acid mine drainage. International Society for Microbial Ecology 7: 1038-1050. 
466 Lauber CL, Hamady M, Knight R, Fierer N (2009) Pyrosequencing-based assessment of soil pH 467 as a predictor of soil bacterial community structure at the continental scale. Applied and Environmental Microbiology 75: 5111-5120.

469

470

471

472

473

474

475

476

477

478

479

480

481

482

483

484

485

486

Lewin A, Wentzel A, Valla, S (2013) Metagenomics of microbial life in extreme temperature environments. Current Opinion in Biotechnology 24: 516-525.

Martin LJ, Adams RI, Bateman A, Bik HM, Hawks J, Hird SM, Hughes D, Kembel SW, Kinney K, Kolokotronis SO, Levy G, McClain C, Meadow JF, Medina RF, Mhuireach G, Moreau CS, Munshi-South J, Nichols LM, Palmer C, Popova L, Schal C, Taubel M, Trautwein M, Ugalde JA, Dunn RR. (2015) Evolution of the indoor biome. Trends in Ecology and Evolution 1909:1-10.

Maruyama A, Taniguchi R, Tanaka H, Ishiwata H, Higashihara T (1997) Low-temperature adaptation of deep-sea bacteria isolated from the Japan Trench. Marine biology 128: 705711.

Mergeay M, Monchy S, Vallaeys T, Auquier V, Benotmane A, Bertin P, Taghavi S, Dunn J, van der Lelie D, Wattiez R (2003) Ralstonia metallidurans, a bacterium specifically adapted to toxic metals: towards a catalogue of metal responsive genes. FEMS Microbiology Reviews 27: $385-410$.

Niehaus F, Bertolodo C, Kähler M, Antranikian G (1999) Extremophiles as a source of novel enzymes for industrial application. Applied Microbiology and Biotechnology 51:711-729.

Noda S, Kitade O, Inoue T, Kawi M, Kanuka M, Hiroshima K, Hongoh Y, Constantino R, UysV, Zhong J, Kudo T, Ohkuma M (2007) Cospeciation in the triplex symbiosis of 
487

488

489

490

491

492

493

494

495

496

497

498

499

500

501

502

503

504

505

506

termite gut protists (Pseudotrichonympha spp.), their hosts, and their bacterial endosymbionts. Molecular Ecology 16:1257-1266.

Oshima K, Hayashi J, Toh H, Nakano A, Shindo C, Komiya K, Morita H, Honda K, Hattori M (2015) Complete genome sequence of Parascardovia denticolens JCM 12538 ${ }^{\mathrm{T}}$, isolated from human dental caries. Genome Announcements 3(3): e00485-15.

Rattanasomboom N, Bellara SR, Harding CL, Fryer PJ, Thomas CR, Al-Rubeai M, McFarlane CM (1999) Growth and enumeration of the meat spoilage bacterium Brochothrix thermosphacta. International Journal of Food Microbiology 51: 145-158.

Rothschild LJ and Mancinelli RL (2001) Life in extreme environments. Nature 409: 1092-1101.

Sharp CE, Brady AL, Sharp GH, Grasby SE, Stott MB, Dunfield PF (2014) Humboldt's spa: microbial diversity is controlled by temperature in geothermal environments. ISME Journal 8: 1166-1174.

Szewzyk U, Szewzyk R, Manz W, Schleifer KH (2000) Microbiological safety of drinking water. Annual Review of Mircrobiology 54: 81-127.

Tringe SF, Zhang T, Liu X, Yu Y, Lee WH, Yap J, Yao F, Suan ST, Ing SK, Haynes M, Rohwer F, Wei CL, Tan P, Bristow J, Rubin EM, Ruan Y (2008) The airborne metagenome in an indoor urban environment. PLoS One 3(4): e1862.

Tripathi BM, Kim M, Singh D, Lee-Cruz L, Lai-Hoe A, Ainuddin, AN, Go R, Rahim RA, Husni MHA, Chun J, Adams JM (2013) Tropical soil bacterial communities in Malaysia: pH dominates in the equatorial tropics too. Microbial Ecology 64: 474-484. 
507 Uchibori S, Tsuzukibashi O, Kobayashi T, Aida M (2012) Localization of the genus Rothia in 508 the oral cavity. International Journal of Oral-Medical Science 11: 207-210.

509 Urban JM, Fergus DJ, Savage, AM, Ehlers M, Menninger HL, Dunn RR, Horvath JE (2016) The 510 effect of habitual antiperspirant and deodorant product use on the armpit microbiome. PeerJ $511 \quad 4: \mathrm{e} 1605$.

512 Valentine DL (2007) Adaptations to energy stress dictate the ecology and evolution of Archea.

$513 \quad$ Nature Reviews Microbiology 5:316-323.

514 Vandekerckhove, TTM, Willems A, Gillis M, Coomans A (2000) Occurrence of novel

515 verrucomicrobial species endosymbiotic and associated with parthenogenesis in Xiphinema 516 americanum-group species (Nematoda, Longidoridae). International Journal of Systematic 517 and Evolutionary Microbiology 50: 2197-2205.

518 van den Burg B (2003) Extremophiles as a source of novel enzymes. Current Opinion in $519 \quad$ Microbiology 6: 213-218.

520 Vannini C, Pöck1 M, Petroni G, Wu QL, Lang E, Stackebrandt E, Schrallhammer M, Richardson

521 PM, Hahn MW (2007) Endosymbiosis in Statu nascendi: close phylogenetic relationship

522 between obligately endosymbiotic and obligately free-living Polyncleobacter strains

523 (Betaproteobacteria). Environmental Microbiology 9: 347-359.

524 Vaz-Moreira I, Nunes OC, Manaia, CM (2011) Diversity and antibiotic resistance patterns of

525 Sphingomonadaceae isolates from drinking water. Applied and Environmental

$526 \quad$ Microbiology 77: 5697-5706. 
527 Wang Q, Garrity GM, Tiedje JM, Cole JR (2007) Naı”ve Bayesian Classifier for Rapid

528 Assignment of rRNA Sequences into the New Bacterial Taxonomy. Applied And

$529 \quad$ Environmental Microbiology 73: 5261-5267.

530 Wang N, Ding L-J, Xu H-J, Li H-B, Su J-Q, Zhu Y-G In Press Variability in responses of

531 bacterial communities and Nitrogen Oxide emission to Urea fertilization among various

$532 \quad$ flooded paddy soils. FEMS Microbiology and Ecology.

533 Wrighton KC, Agbo P, Warnecke F, Weber KA, Brodie EL, DeSantis, TZ, Hugenholtz P, 534 Andersen GL, Coates JD (2008) A novel ecological role of the Firmicutes identified in 535 thermophilic microbial fuel cells. ISME Journal 2: 1146-1156.

536 Yi H, Yoon HI, Chun J (2005) Sejongia antarctica gen. nov., sp. nov. and Sejongia jeonii sp. 537 nov., isolated from the Antarctic. 55: 409-416.

\section{Figure Captions:}

539 Figure 1: OTU accumulation curves for each extreme environment, expressed as number of

540 OTUs by number of reads from sequencing. Each curve was constructed using 1000 iterations,

541 and the dotted lines represent 95\% confidence intervals. Non-overlapping confidence intervals

542 indicate that the accumulation curves are significantly different. Thus, habitats with extreme

543 temperatures had significantly more accumulated species than habitats with either extreme $\mathrm{pH}$ or

544 extreme chemical environments. However, the accumulated species in habitats $\mathrm{pH}$ and chemical

545 extremes did not differ significantly.

546 Figure 2: Comparison of rarefaction curves between extreme and non-extreme habitats. (A)

547 extreme $v s$. intermediate temperatures, (B) extreme $v s$. neutral $\mathrm{pH}$ environments, and (C)

548 extreme chemicals present $v s$. absent. Rarefaction curves are expressed as number of OTU by 
549 number of reads from sequencing. Each curve was constructed using 1000 iterations, and the

550 dotted lines represent 95\% confidence intervals. Significance tests were as described for Figure 5511.

552 Figure 3: NMDS ordinations OTU occurrence by (A-B) Temperature \& pH and (C-D)

553 Temperature \& chemical environments in the home. Large symbols represent centroids $\pm 1 \mathrm{SE}$

554 (A, C), and small symbols represent each sample (B, D). The interaction between temperature 555 and $\mathrm{pH}$ was significant (PERMANOVA: $\left(\right.$ pseudo) $\left.-\mathrm{F}_{1,85}=2.53, P(\mathrm{perm})=0.0001\right)$, as was the 556 interaction between extreme temperature and chemical conditions (PERMANOVA: (pseudo)- $\mathrm{F}_{1}$, $55785=3.16, P($ perm $)=0.0001)$. The ordination was constructed with Bray-Curtis distances and 100 558 restarts; 2-D stress was 0.21. PERMANOVA analyses were conducted using type III sums of 559 squares and 9,999 iterations.

560 Figure 4: Average distances between samples and centroids ( $\beta$-diversity) across home

561 environments that differ with respect to extreme temperatures and (A) extreme $\mathrm{pH}$ conditions \&

562 (B) extreme chemical conditions. Data were assessed using PermDisp; dispersion was

563 significantly different across extreme temperatures and extreme $\mathrm{pH}$ conditions $\left(\mathrm{F}_{3,82}=4.08\right.$, $564 P=0.024)$ and across extreme temperatures and extreme chemical conditions $\left(\mathrm{F}_{3,82}=6.99\right.$, $565 P=0.0017)$. Post-hoc pairwise tests: $* P<0.025, * * \mathrm{P}<0.01, * * * \mathrm{P}<0.001$.

\section{Table Captions:}

567 Table 1: Summary of occurrences of microbes that were present in samples from extreme home 568 environments, but absent from the broader home samples. Numbers indicate the number of reads 569 of each genus by extreme environment. The first group includes genera that were only present in 570 one extreme environment, the second group includes genera that were present in two extreme 
571 environments, and the last group includes genera that were present in all three extreme home

572 environments.

573 Supplementary Tables and Figures:

574 Raw Data: Output file from QIIME at the genus level (L6), rarefied to 1000 reads.

575 Supp. Table 1: Description of sample locations. Standardized locations were sampled in all 6

576 houses, while special locations were only sampled in a subset of the houses (due to availability

577 of samples across houses)

578

579 Supp. Table 2: Classifications of sampled extreme home environments based upon temperature, $580 \mathrm{pH}$ and chemical conditions.

581 Supp. Table 3:_Results from PERMANOVAs testing (a) the effects of house ID on microbial

582 composition; and (b) the effects of temperature class, $\mathrm{pH}$ class, and house ID on microbial

583 composition.

584 Supp. Table 4: List of non-human associated microbes in extreme and non-extreme (Dunn et al. 585 2013) home habitats

586

587 Supp. Figure 1: Map of houses that were sampled for the study

588 Supp. Figure 2: NMDS plot with houses and sampling locations labeled.

589 Supp. Figure 3: Histograms depicting the \% of reads from bacterial and archaeal classes in (a) 590 habitats with extreme temperatures, (b) habitats with extreme $\mathrm{pH}$, and (c) habitats with extreme 591 chemicals.

592

593

594

595 


\section{Table $\mathbf{1}$ (on next page)}

Table 1

Summary of occurrences of microbes that were present in samples from extreme home environments, but absent from the broader home samples. Each $\mathrm{X}$ indicates that the genus was found in a given extreme environment. 
1

Table 1: Summary of occurrences of microbes that were present in samples from extreme home

3 environments, but absent from the broader home samples. Numbers indicate the number of reads of each

4 genus by extreme environment. The first group includes genera that were only present in one extreme

5 environment, the second group includes genera that were present in two extreme environments, and the

6 last group includes genera that were present in all three extreme home environments.

7

8

9

10

11

12

\begin{tabular}{|l|c|c|c|}
\hline \multicolumn{1}{|c|}{ Genus } & $\begin{array}{c}\text { Extreme } \\
\text { Temperatures }\end{array}$ & $\begin{array}{c}\text { Extreme } \\
\mathbf{p H}\end{array}$ & $\begin{array}{c}\text { Extreme } \\
\text { Chemical }\end{array}$ \\
\hline Brochothrix & 265 & 0 & 0 \\
\hline Buchnera & 22 & 0 & 0 \\
\hline Polynucleobacter & 33 & 0 & 0 \\
\hline Ralstonia & 21 & 0 & 0 \\
\hline Thermicanus & 34 & 0 & 0 \\
\hline Helcococcus & 0 & 0 & 22 \\
\hline \multicolumn{2}{|r|}{}
\end{tabular}

\begin{tabular}{|l|c|c|c|}
\hline Solibacter & 86 & 0 & 30 \\
\hline Brevundimonas & 184 & 189 & 0 \\
\hline Azobacteroides & 0 & 33 & 33 \\
\hline Elizabethkingia & 0 & 25 & 24 \\
\hline Xiphinematobacter & 0 & 19 & 21 \\
\hline
\end{tabular}

\begin{tabular}{|l|c|c|c|}
\hline Azospira & 139 & 33 & 44 \\
\hline Brachybacterium & 101 & 52 & 69 \\
\hline Enhydrobacter & 452 & 387 & 408 \\
\hline Gluconobacter & 23 & 21 & 22 \\
\hline Oligella & 40 & 74 & 77 \\
\hline Parascardovia & 141 & 46 & 107 \\
\hline Photobacterium & 71 & 65 & 93 \\
\hline Propionibacterium & 73 & 31 & 40 \\
\hline Salinibacterium & 108 & 334 & 355 \\
\hline
\end{tabular}




\section{Figure 1}

OTU accumulation curves for each extreme environment

OTU accumulation curves for each extreme environment, expressed as the number of OTUs by the number of reads from sequencing. Each curve was constructed using 1000 iterations, and the dotted lines represent 95\% confidence intervals. Non-overlapping confidence intervals indicate that the accumulation curves are significantly different. Thus, habitats with extreme temperatures had significantly more accumulated species than habitats with either extreme $\mathrm{pH}$ or extreme chemical environments. However, the accumulated species in habitats $\mathrm{pH}$ and chemical extremes did not differ significantly.

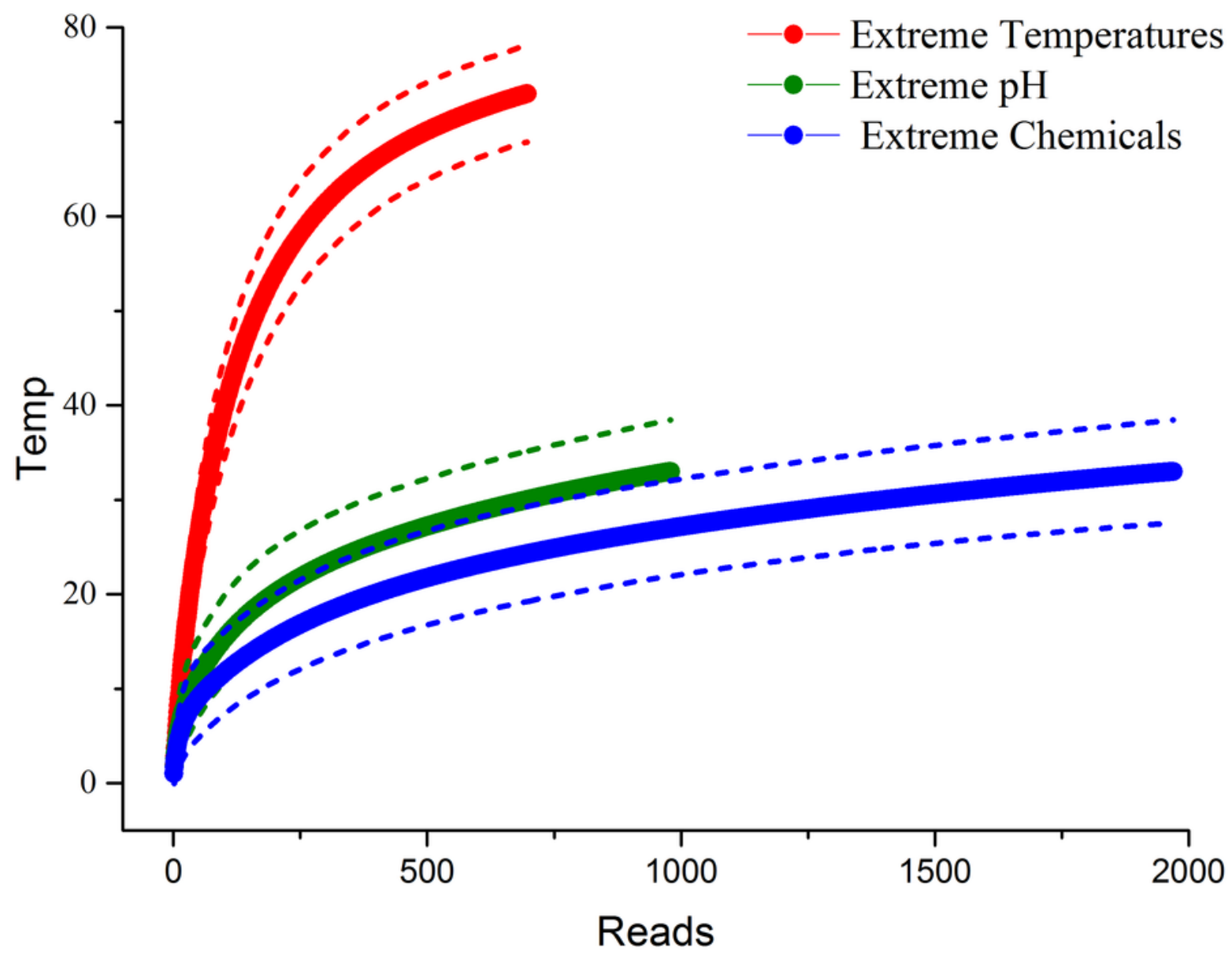




\section{Figure 2}

Comparison of rarefaction curves between extreme and non-extreme habitats

Comparison of rarefaction curves between extreme and non-extreme habitats . (A) extreme vs. intermediate temperatures, (B) extreme vs. neutral pH environments, and (C) extreme chemicals present vs. absent. Rarefaction curves are expressed as number of OTU by number of reads from sequencing. Each curve was constructed using 1000 iterations, and the dotted lines represent $95 \%$ confidence intervals. Significance tests were as described for Figure 1. 


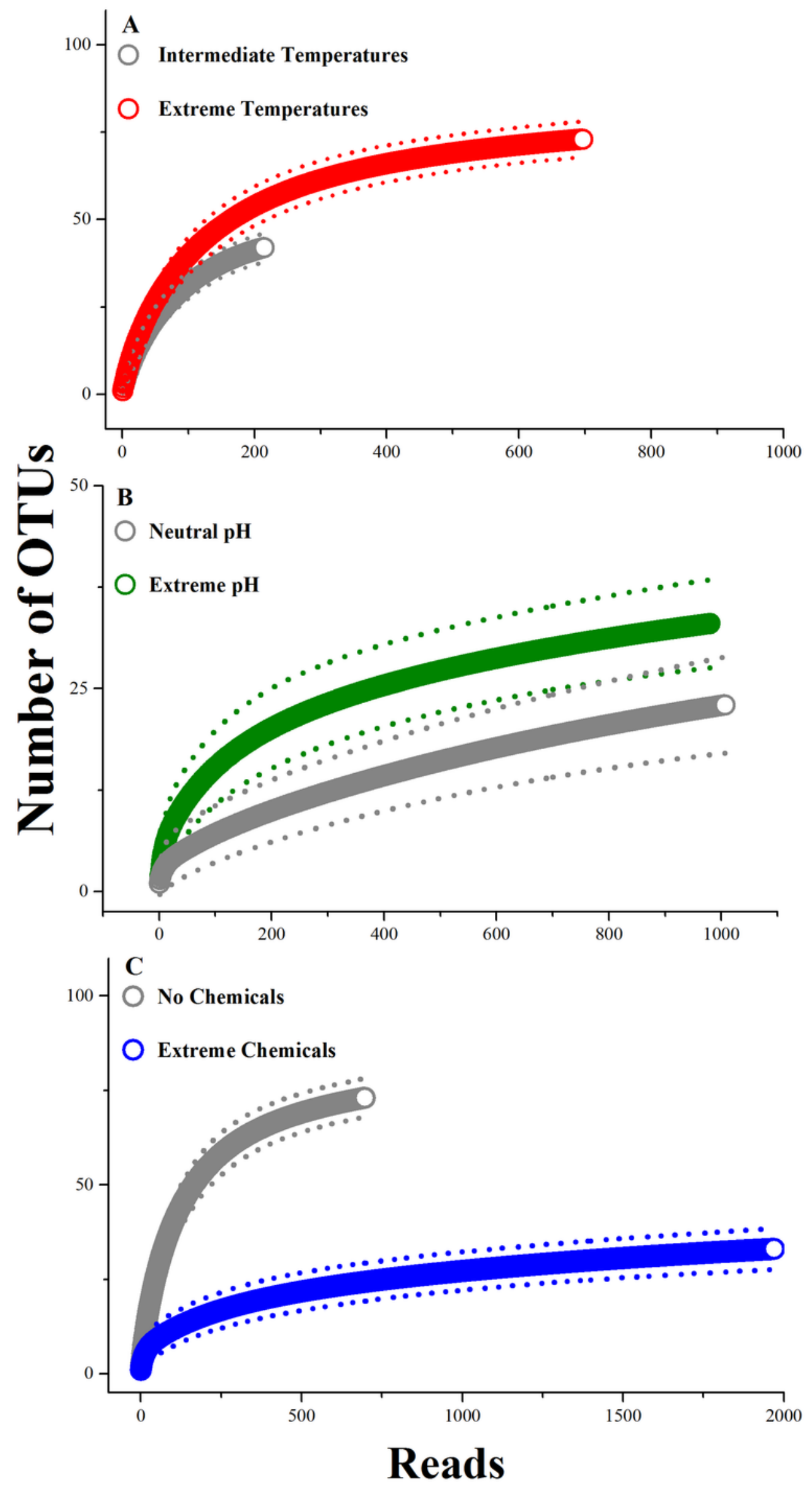


Figure 3

NMDS ordinations OTU occurrence by (A-B) Temperature \& pH and (C-D) Temperature \& chemical environments in the home.

NMDS ordinations OTU occurrence by (A-B) Temperature $\&$ pH and (C-D) Temperature \& chemical environments in the home. Large symbols represent centroids $\pm 1 \mathrm{SE}(\mathrm{A}, \mathrm{C})$, and small symbols represent each sample $(B, D)$. The interaction between temperature and $\mathrm{pH}$ was significant (PERMANOVA: (pseudo) $-\mathrm{F}_{1,85}=2.53, P($ perm $)=0.0001$ ), as was the interaction between extreme temperature and chemical conditions (PERMANOVA: (pseudo) $-\mathrm{F}_{1,85}=3.16, P($ perm $\left.)=0.0001\right)$. The ordination was constructed with Bray-Curtis distances and 100 restarts; 2-D stress was 0.21 . PERMANOVA analyses were conducted using type III sums of squares and 9,999 iterations.
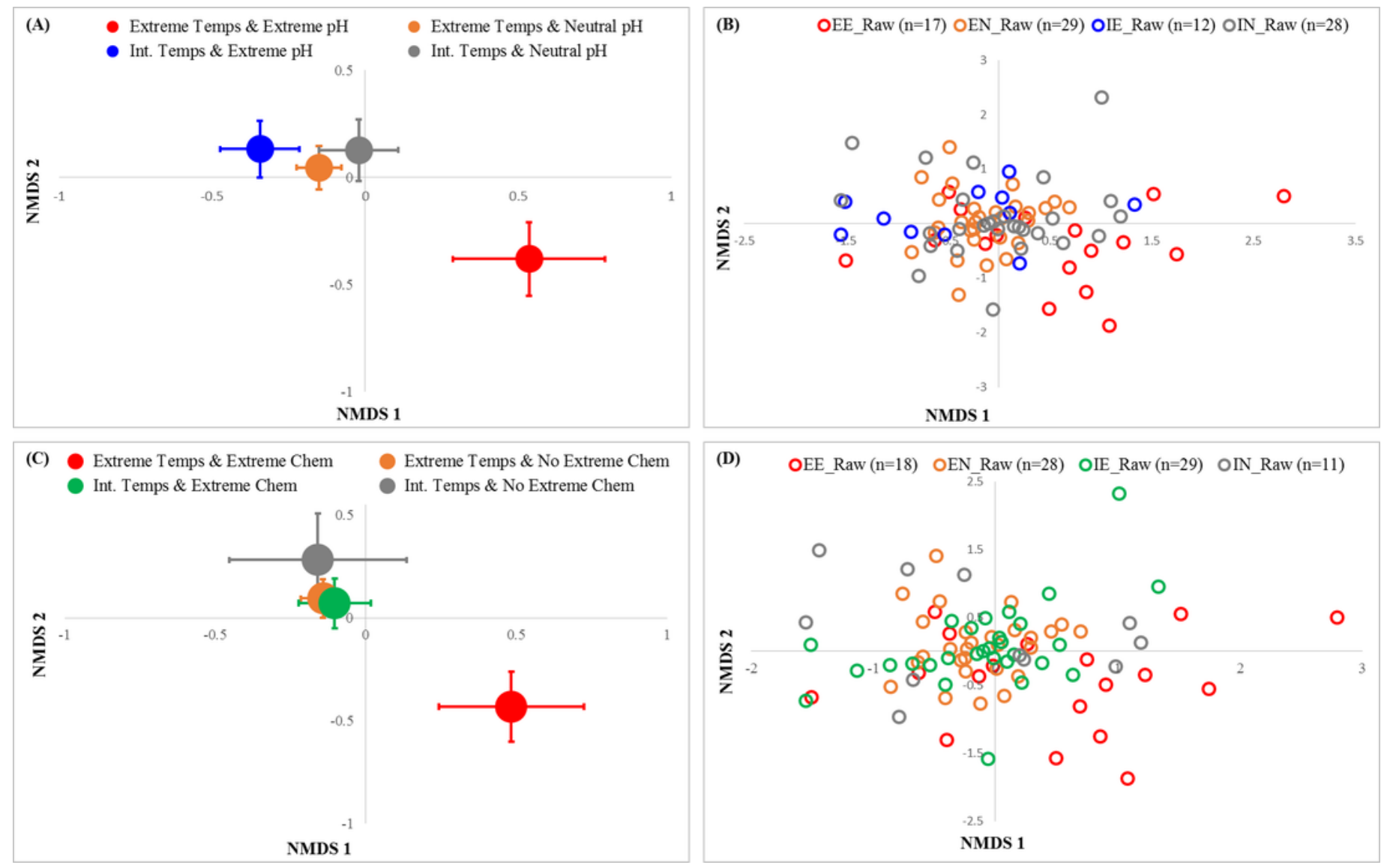


\section{Figure 4}

Average distances between samples and centroids ( $\beta$-diversity) across home environments

Average distances between samples and centroids ( $\beta$-diversity) across home environments that differ with respect to extreme temperatures and $(A)$ extreme $\mathrm{pH}$ conditions \& $(B)$ extreme chemical conditions. Data were assessed using PermDisp; dispersion was significantly different across extreme temperatures and extreme $\mathrm{pH}$ conditions $\left(\mathrm{F}_{3,82}=4.08\right.$, $P=0.024)$ and across extreme temperatures and extreme chemical conditions $\left(F_{3,82}=6.99\right.$, $P=0.0017)$. Post-hoc pairwise tests: $* P<0.025, * * P<0.01, * * * P<0.001$.
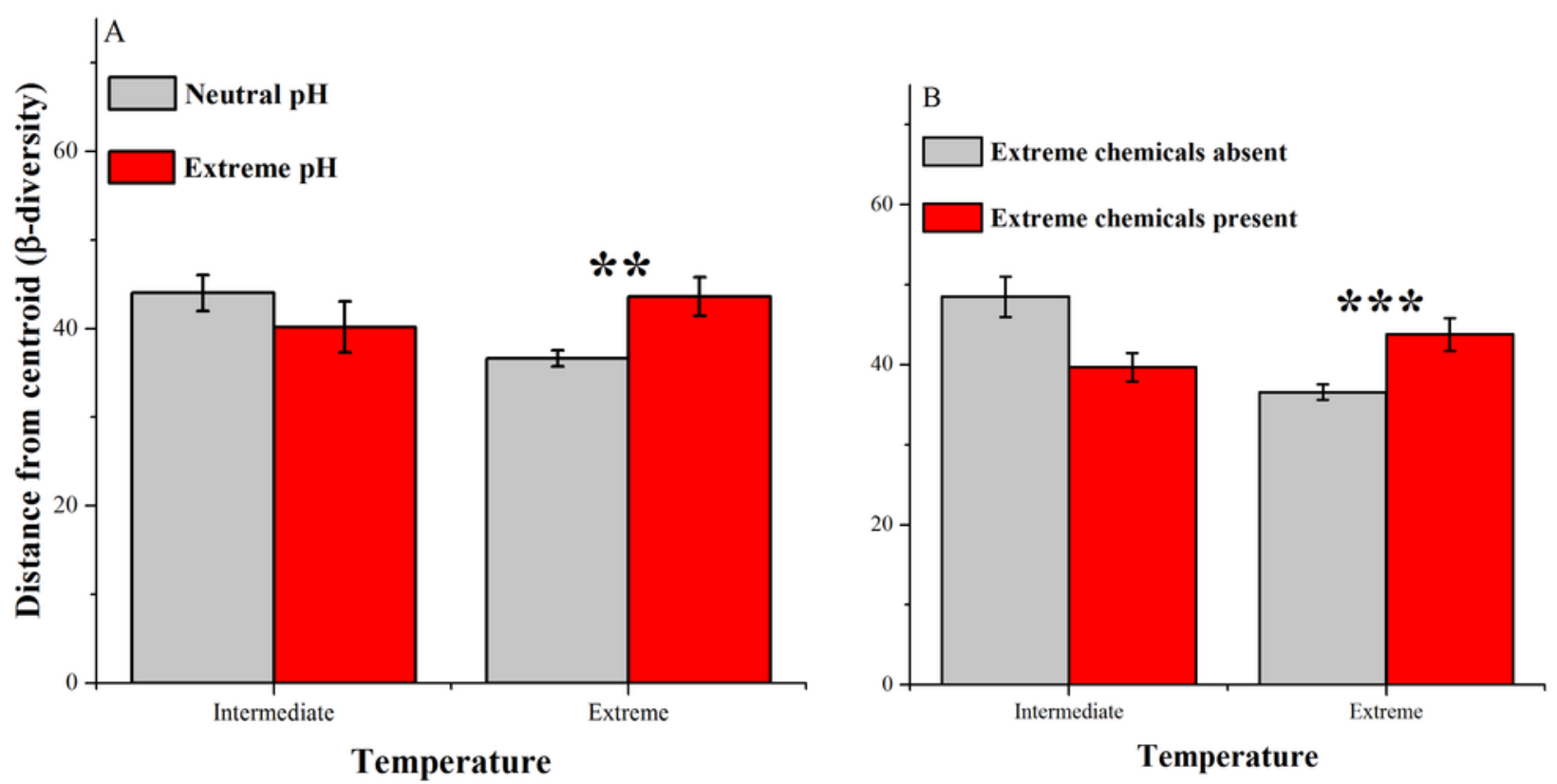